\title{
Erratum to: Pattern of resting site of sables in Daxing'an Mountains
}

(C) Northeast Forestry University and Springer-Verlag Berlin Heidelberg 2011

Retraction: XU Cheng-shui, ZOU Hong-fei, ZHANG Hong-hai. Pattern of resting site of sables in Daxing'an Mountains. Journal of Forestry Research 2000, 11(2): 123-126. DOI: 10.1007/BF02856689

This article is retracted because of copyright violation. 